\title{
INDEX OF VOLUME 57
}

Subjects:

\section{INDEX OF ABSTRACTS}

Algebra and Theory of Numbers, 64, 76, 118, 167, 175, 251, 265, 284, 297, 367, 457.

Analysis, 59, 66, 78, 123, 168, 176, 253, 269, 286, 300, 370, 463.

Applied Mathematics, 60, 71, 135, 170, 182, 277, 291, 303, 479.

Geometry, 72, 81, 139, 170, 184, 254, 278, 291, 483.

Logic and Foundations, $81,255,485$.

Statistics and Probability, 73, 82, 141, 171, 184, 279, 292, 305, 372, 486.

Topology, 62, 74, 82, 141, 172, 185, 256, 280, 293, 305, 372, 487.

Authors:

Agmon, S., 463; Ainsworth, O. R., 135; Aissen, M. I., 59, 176; Akutowicz, E. J., 71; Albers, L. U., 66; Albert, A. A., 167, 457, 457; Amitsur, S. A., 118; Anderson, R. D., 185; Andrews, J. J., 73; Antosiewicz, H. A., 123; Apostol, T. M., 76, 370, 370; Arens, R., 487; Arf, C., 136, 269; Arnold, B. H., 305; Aronszajn, N., 124, 124, 286, 286.

Bade, W. G., 78; Barsotti, I., 184; Bauer, W. F., 479; Baum, W. R., 141, 172; Becker, H. W., 297, 298; Bergman, S., 177, 177, 184, 270; Berkovitz, L. D., 287; Bernardi, S. D., 177, 178; Bers, L., 124, 463, 463; Bing, R. H., 293, 293, 487; Birnbaum, Z. W., 305; Blakers, A. L., 62, 141, 142, 142, 142, 143; Boas, R. P., 67; Bonsall, F. F., 125; Bose, R. C., 265; Bott, R., 136, 143, 280; Botts, T. A., 367; Bourgin, D. G., 463; Boyer, J. M., 125; Brand, L., 170; Brauer, A. T., 251, 266; Brenner, J. L., 368; Breusch, R. H., 270; Brock, P., 270; Browder, F., 270, 280, 280; Brownell, F. H., 79, 300; Bruck, R. H., 64; Brunk, H. D., 300; Buck, R. C., 286; Burgess, C. E., 487, 488; Burton, L. P., 253; Bush, K. A., 265, 266, 266, 271.

Calabi, E., 254, 291, 488; Calder6n, A. P., 464; Cameron, R. H., 286; Carlitz, L., 266, 457, 457, 457; Carr, R. E., 464; Carruth, P. W., 298; Cesari, L., 67, 67, 168, 465; Charnes, A., 480; Chatland, H., 458; Chehata, C. G., 251; Chen, K. T., 143, 488; Cherlin, G. Y., 271; Chernoff, H., 67; Chowla, S., 125; Chu, J. T., 68; Chung, K. L., 141, 279; Clarke, A. B., 267; Clement, M. D., 292; Coburn, N., 136; Cohen, E., 119, 175, 458; Cohn, H., 64, 65, 119, 175, 287, 458; Copeland, A. H., 125; Cowling, V. F., 271, 465; Coxeter, H. S. M., 255, 278; Curtis, C. W., 267; Curtis, M. L., 74, 293; Curtiss, J. H., 73.

Davis, A. C., 77; Davis, E. A., 303; DeCicco, J., 139, 139; Dekker, J. C. E., 119; Devinatz, A., 68; De Vogelaere, R., 182; Diaz, J. B., 271, 272; Dieudonné, J. A., 272; Dilworth, R. P., 65; Dolph, C. L., 480, Douglas, J., 267, 458; Dressel, F. G., 179; Duffin, R. J., 136; Duncan, D. G., 77; Durst, L. K., 298; Du Val, P., 139; Dye, H. A., 79.

Eaves, J. C., 119; Eckmann, B., 488; Edrei, A., 465 ; Eilenberg, S., 294; Eisenstadt, B. J., 465; Ellis, D. O., 120, 125, 143, 144, 170, 255, 256, 483; Estill, M. E., 281, 488; Evans, R. L., 287.

Federer, H., 272, 273; Feibleman, J. K., 255; Fialkow, A. D., 182, 183; Finn, R. S., 178, 273, 466; Flanders, H., 77; Floyd, E. E., 144, 144, 489, 489; Forsythe, G. E., 183, 304, 304, 480, 481; Fort, M. K., 74; Fortet, R. E., 171, 171, 171, 172; Foster, A. E., 459; Fox, C., 126; Frame, J. S., 120, 459; Frank, E., 466; Freilich, G., 268; Friberg, M. S., 466; Fuglede, B., 370, 466; Fulkerson, D. R., 368; Fullerton, R. E., 67.

Gaddum, J. W., 292; Gaffney, M. P., 68; Gaier, D., 178; Gale, D., 175; Garabedian, P. R., 467, 467; Gelbaum, B. R., 126, 467; Gergen, J. J., 179; Gerst, I., 182, 
183; Ghaffari, A., 481, 486; Gilbarg, D., 137; Gillman, L., 459; Givens, W., 459; Goffman, C., 468; Goheen, H. E., 179; Gokhale, V. D., 120; Goldman, O., 176; Gomory, R., 60; Goodman, A. W., 126, 468; Gordon, W. L., 256; Gorciu, V. G., 489; Gosselin, R. P., 287; Green, J. W., 370; Greenberg, H. J., 480.

Hadlock, E. H., 121; Haimo, F., 65, 121, 460; Hall, D. W., 147; Halmos, P. R., 294, 468; Halperin, I., 126; Hammer, P. C., 127, 278, 484, 484, 485; Harary, F., 125; Harrold, O.G., 489; Hatfield, C., 286; Heerema, N., 460; Heins, M. H., 127, 127; Henriksen, M., 284; Herriot, J. G., 300; Herstein, I. N., 284; Herzog, F., 69; Hestenes, M. R., 291, 480; Hewitt, E., 128, 303; Higman, D. G., 284; Hille, E., 468, 469, 469; Hodge, P. G., Jr., 481; Hoffman, A. J., 140, 279; Holyoke, T. C., 121; Horn, A., 79; Householder, A. S., 69; Hu, S. T., 62, 82, 83, 83, 256, 372, 373, 490, 490; Hua, L. K., 121; Hutcherson, W. R., 484; Hyers, D. H., 300.

Ingersoll, B. M., 273; Isaacs, R., 80; Ito, W. H., 469.

Jackson, J. R., 490; Jacobson, N., 460; James, R. C., 128; Jamison, S. L., 128; Jennings, A. K., 286; Jerison, M., 469; John, F., 301; Johnson, R. E., 268; Jónsson, B., 168; Jones, B. W., 122; Jones, F. B., 144.

Kadison, R. V., 129, 179, 370, 466; Kakutani, S., 491; Kalisch, G. K., 467; Kampé de Fériet, J., 129; Kanter, L. H., 69; Kaplan, W., 287; Kaplansky, I., 461; Karp, S. N., 60; Karush, W., 291; Katz, S., 176; Kelley, J. L., 257, 470; Kiefer, J. C., 372; Klamkin, M. S., 59, 129; Klee, V. L., 470; Kleene, S. C., 485; Klein, G., 288; Kleinfeld, E., 285; Koenig, J. F., 168; Krzywoblocki, M. Z., 71, 291.

Lanczos, C., 301; Lang, G. B., 256; Lax, P. D., 179, 274; Leipnik, R. B., 80, 301; Lepson, B., 59, 180, 274; Leutert, W. W., 61; Levit, R. J., 130; Levitzki, J., 122, 268; Lewy, H., 301; Liao, S. D., 373; Lohwater, A. J., 69, 130, 470, 470; Lonseth, A. T., 371 ; Loomis, L. H., 122; Lorch, L., 288; Lorentz, G., 126; Lowney, R. E., 70; Ludford, G. S. S., 277; Lyndon, R. C., 269.

McArthur, C. W., 256; McGregor, J. L., 371; McKinsey, J. C. C., 485; McLaughlin, J. E., 65; McShane, E. J., 371; Macauley, R. A., 368; MacLane, G. R., 471; MacLane, S., 144, 294; Mancill, J. D., 253; Mann, H. B., 169, 368, 458, 461; Marden, M., 125; Marsaglia, G., 371; Marx, I., 471; Massey, W. S., 62, 74, 141, 142, 142, 142, 281, 281, 491, 491; Melvin, M. A., 369; Milgram, A. N., 59, 130; Miller, C. E., 492; Miller, K. S., 131, 253, 372; Mitchell, Benjamin Ernest, 140, 140, 255; Mitchell, J. M., 131, 288; Moise, E. E., 75, 492, 492; Montgomery, D., 75, 145; Moore, C. N., 131; Mordell, L. J., 461; Morrison, D. R., 251; Moser, L., 461; Mostow, G. D., 122, 131, 145; Motzkin, T. S., 183, 302, 304, 471, 481; Muhly, H. T., 65; Mulholland, H. P., 486; Murray, F. J., 61, 270.

Nehari, Z., 131; Nemmers, F. E., 66; Nering, E. D., 285; Newman, D. J., 471, 472; Newman, J. J., 80; Nikodým, O. M., 493; Niven, I., 298; Norris, M. J., 493, 493; Novikoff, A., 274; Novosad, R. S., 257.

Oliver, H. W., 472, 472; Ostrom, T. G., 461; Owens, O. G., 70.

Paige, L. J., 77, 369; Pardee, O. O., 137; Parker, W. V., 251; Peeples, W. D., 252; Peters, G. O., 132; Pettis, B. J., 254, 257, 257, 473; Pfeiffer, P. E., 473; Phillips, R. S., 274; Piranian, D., 473; Piranian, G., 69, 69, 271, 289, 471, 473; Pitcher, E., 474, 493; Polansky, F. J., 474; Price, G. B., 66.

Rabinowitz, P., 282; Rabson, G., 474; Rad6, T., 294; Raney, G. N., 369; Ratner, L. T., 474; Reade, M. O., 132, 475; Rechard, O. W., 289; Rees, P. K., 132; Reeves, R. F., 289; Reich, E., 82; Reichelderfer, P. V., 172; Reiner, I., 121; Resch, D., 291; Reynolds, T. L., 266; Richmond, D. E., 60; Rickart, C. E., 460; Roberts, R. C., 272; Robertson, M. S., 275; Robinson, L. B., 180, 180, 372; Rosenbaum, I., 170, 279; Rosenberg, A., 285; Rosenblatt, M., 292, 486; Rosenbloom, P. C., 59, 130, 475; 
Rosenlicht, M., 269; Rosenthal, J. E., 183; Rosser, J. B., 480; Rothe, E. H., 295; Rothe, J. C., 75; Rudin, W., 180, 181, 475; Rutledge, W. A., 123.

Salzer, H. E., 72, 137; Sanders, J., 181, 275; Sario, L. R., 275, 276, 475; Schafer, R. D., 269; Scherk, P., 462, 462; Schild, A., 276; Schoenberg, I. J., 484; Schwartz, J. T., 476, 476; Schweitzer, A. R., 70, 72; Scott, W. R., 285; Seiden, E., 72; Shanks, M. E., 75, 169, 295; Sherman, S., 476; Silva, J. A., 252, 269; Silverman, E., 289; Sinclair, A., 290; Singer, I. M., 60, 476; Slater, M. L., 477; Smiley, M. F., 462; Smith, C. B., 138; Snapper, E., 299; Snell, J. L., 486; Sobczyk, A., 127, 278, 482, 484, 484, 485; Sopka, J. J., 133; Spencer, D. C., 254, 467; Spencer, D. E., 61; Spencer, S. M., 252; Stein, M. L., 302, 482; Stewart, F. M., 175, 181; Straus, E. G., 306; Sugar, A. C., 485; Sunyer i Balaguer, F., 181; Suppes, P., 485; Szász, O., 133, 169, 290.

Talmadge, R. B., 306; Tanimoto, T. T., 133; Tarski, A., 78, 81, 81; Taussky, O., 123; Taylor, H. E., 133; Taylor, R. L., 145, 145, 146, 146, 146, 147; Thomas, G. H. M., 295, 494; Thomas J. M., 254; Thompson, F. B., 82; Thompson, J. E. 477; Thurston, H. S., 252; Tingey, F. H., 305; Titt, E. W., 276; Todd, J., 123; Tornheim, L., 463, 478; Tricomi, F. G., 80, 134, 141, 302, 305; Trombley, E. F., 71; Truesdell, C. A., 138; Turrittin, H. L., 290.

Ulam, S. M., 300; Utz, W. R., 66, 494.

Valentine, F. A., 81, 306; Vanderslice, Mrs. B. R., 147; Van Tuyl, A. H., 138; Verblunsky, S., 140; Vezeau, W. A., 73; Vinograde, B., 463.

Wade, T. L., 123; Wall, H. S., 134; Wallace, A. D., 147, 252, 258; Walsh, J. L., 471, 478; Wang, H. C., 258, 478; Ward, L. E., Jr., 258; Ward, M., 78, 299; Wasow, W. R., 184; Waterman, D. R., 71; Weinberger, H. F., 134, 182, 482; Weinstein, A., 134, 138, 277, 483; Wendel, J. G., 135, 478; White, P. A., 306; Whitehead, G. W., 491, 491; Whitehead, J. H. C., 144; Whiteman, A. L., 299; Whyburn, W. M., 253; Widder, D. V., 277; Wiegmann, N. A., 66; Wilansky, A., 182; Wilder, R. L., 295; Wolf, F., 303; Wolfowitz, J., 279; Wong, Y. K., 277; Wyler, O., 296.

Yang, C. T., 258; Yood, B., 479; Yosida, K., 303; Young, D. M., 478; Young, F. H., 81; Young, G. S., 75, 293; Yu, C. Y., 479.

Zadeh, L. A., 61, 278, 279, 483; Zeichner, A., 124; Zelinsky, D., 285; Ziebur, A. D., 135; Zimmerberg, H. J., 135; Zippin, L., 75, 145; Zuckerman, H. S., 76, 128; Zygmund, A., 464.

\section{INDEX OF REPORTS OF MEETINGS AND MISCELLANEOUS ARTICLES}

Brahana, H. R., George Abram Miller, 1863-1951, 377.

Cohen, L. W. Reports of meetings of the American Mathematical Society: February Meeting in New York, 174; April Meeting in New York, 259.

Green, J. W., Reports of meetings of the American Mathematical Society: November Meeting in Los Angeles, 76; April Meeting in Stanford, 297; June Meeting in Pullman, 367.

Hollcroft, T. R., Reports of meetings of the American Mathematical Society: October Meeting in New York, 55.

Kline, J. R., The International Congress of Mathematicians, 1.

Lorch, E. R. Joseph Fels Ritt, 307.

Martin, M. H., and Whyburn, W. M. Fourth Symposium on Applied Mathematics, 374.

Miller, G. A. See Brahana, H. R.

Ritt, J. F. See Lorch, E. R.

Whyburn, W. M. Reports of meetings of the American Mathematical Society: 
Annual Meeting of the Society, 109; A pril Meeting in New Orleans, 250.

See Martin, M. H.

Youngs, J. W. T. Reports of meetings of the American Mathematical Society: November Meeting in Evanston, 63; February Meeting in Cincinnati, 167; April Meeting in Chicago, 283; Summer Meeting in Minneapolis, 449.

\section{INDEX OF BOOK REVIEWS}

Ahlfors, L. V. See Schaeffer, A. C.

Allendoerfer, C. B. See Blaschke, W.

Bateman, P. T. See Neville, E. H.

Behnke, H. See Bergman, S.

Bergman, S. Sur la fonction noyau d'un domaine et ses applications dans la théorie du transformations pseudoconformes. $\mathrm{H}$. Behnke, 186.

Bergmann, P. G. See Costa de Beauregard, O.

Bernstein, D. L. Existence theorems in partial differential equations. F. John, 323

Birkhoff, G. Hydrodynamics, a study in logic, fact, and similitude. J. J. Stoker, 497.

Birkhoff, G. D. Collected mathematical papers. 163.

Blaschke, W. Einführung in die Differentialgeometrie. C. B. Allendoerfer, 84.

Blumenthal, L. M. See Segre, B.

Bochner, S. See Cinquini, S., Zygmund, A.

Bol, G. Projektive Differentialgeometrie. V. Dalla Volta, 398.

Bompiani, E. See Struik, D. J.

Bremmer, H. See van der Pol, B.

Calderón, A. P. See Zygmund, A.

Carathéodory, C. Funktionentheorie. M. Heins, 190.

Chandrasekharan, K. See Denjoy, A.

Chevalley, C. Introduction to the theory of algebraic functions of one variable. A. Weil, 384.

Cinquini, S. Funzioni quasi-periodiche. S. Bochner, 331.

Contributions to the theory of games. J. Wolfowitz, 495.

Costa de Beauregard, O. La théorie de la relativité restrainte. P. G. Bergmann, 188.

Courant, R., and Friedrichs, K. O. Supersonic flow and shock waves. C. C. Lin, 85.

Coxeter, H. S. M. See Verriest, G.

Dalla Volta, V. See Bol, G.

Davis, H. T. See Sakellariou, N.

Day, M. M. See Schatten, R.

DeCicco, J. See Synge, J. L.

Denjoy, A. Lecons sur le calcul des coefficients d'une séries trigonométrique. K. Chandrasekharan, 153.

Doob, J. L. See Fréchet, M.

Einstein, A. The meaning of relativity. 207.

Erdélyi, A. See van der Pol, B.

Feller, W. An introduction to probability theory and its applications. Vol. I. J. Wolfowitz, 156.

Fox, C. An introduction to the calculus of variations. L. M. Graves, 207.

Fréchet, M. Généralités sur les probabilités. Éléments aléatoires. J. L. Doob, 206.

Friedrichs, K. O. See Courant, R.

Galois, E. Oeuvres mathématiques d'Évariste Galois publiées en 1897, suivies d'une notice sur Évariste Galois et la théorie des équations algébriques par G. Varriest. 208.

Ghaffari, A. G. The hodograph method in gas dynamics. C. C. Lin, 502.

Graves, L. M. See Fox, C. 
Gröbner, W. Moderne algebraische geometrie. Die idealtheoretischen Grundlagen. A. Seidenberg, 87.

Halmos, P. R. See Tietze, H.

Hamel, G. Theoretische Mechanik. Eine einheitliche Einführung in die gesamte Mechanik. W. Prager, 159.

Heins, M. See Carathéodory, C.

Hochschild, G. See Schilling, O. F. G.

John, F. See Bernstein, D. L.

Jellinek, K. Weltsystem, Weltäther und die Relativitätstheorie. A. Schild, 161.

Kolchin, E. R. See Tschebotaröw, N.

van der Kulk, W. See Schouten, J. A.

Lanczos, C. The variational principles of mechanics. D. C. Lewis, 88

Levi, H. See Walker, R. J.

Lewis, D. C. See Lanczos, C.

Lin, C. C. See Courant, R., Ghaffari, A. G.

MacLane, S. See Pollard, H.

Magnus, W. See Oberhettinger, F.

Marden, M. See Walsh, J. L.

Morse, M. See Zygmund, A.

Neville, E. H. The Farey series of order 1025, displaying solutions of the Diophantine equation $b x-a y=1$. P. T. Bateman, 325 .

Oberhettinger, F., and Magnus, W. Anwendung der elliptischen Funktionen in Physik und Technik. W. Seidel, 204.

van der Pol, B., and Bremmer, H. Operational calculus. A. Erdélyi, 319.

Pollard, H. The theory of algebraic numbers. S. MacLane, 162.

Prager, W. See Hamel, G.

Robbins, H. See Wald, A.

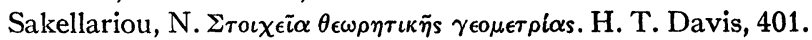

Schaeffer, A. C., and Spencer, D. C. Coefficient regions for schlicht functions. L. V. Ahlfors, 328.

Schatten, R. A theory of cross-spaces. M. M. Day, 326.

Schild, A. See Jellinek, K., Synge, J. L.

Schilling, O. F. G. The theory of valuations. G. Hochschild, 91.

Schouten, J. A., and van der Kulk, W. Pfaff's problem and its generalizations. J. M. Thomas, 94.

Schrödinger, E. Space-time structure. A. H. Taub, 205.

Segre, B. Lezioni de geometria moderna. Vol. I. Fondamenti di geometria sopra un corpo qualsiasi. L. M. Blumenthal, 192.

Seidel, W. See Oberhettinger, F.

Seidenberg, A. See Gröbner, W.

Shohat, J. A., and Tamarkin, J. D. The problem of moments. 332.

Spencer, D. C. See Schaeffer, A. C.

Stoker, J. J. See Birkhoff, G.

Struik, D. J Lectures on classical differential geometry. E. Bompiani, 154.

Synge, J. L., and Schild, A. Tensor calculus. J. DeCicco, 500.

Tamarkin, J. D. See Shohat, J. A.

Taub, A. H. See Schrödinger, E.

The French bibliographical digest. No. 6. 208.

Thomas, J. M. See Schouten, J. A.

Tietze, H. Gelöste und ungelöste mathematische Probleme aus alter und neuer Zeit. P. R. Halmos, 502.

Transue, W. See Zygmund, A. 
Tschebotaröw, N. Grundzilge der Galois'schen Theorie. E. R. Kolchin, 155.

Verriest, G. Introduction à la géométrie non euclidienne par la méthode élémentaire.

H. S. M. Coxeter, 499.

See Galois, E.

van der Waerden, B. L. Modern algebra. Vol. 1. D. Zelinsky, 206.

Modern algebra. Vol. 2. D. Zelinsky, 206.

Moderne Algebra, Vol. 1. D. Zelinsky, 206.

Wald, A. Statistical decision functions. H. Robbins, 383.

Walker, R. J. Algebraic curves. H. Levi, 160.

Walsh, J. L. The location of critical points of analytic and harmonic functions. M. Marden, 194.

Weil, A. See Chevalley, C.

Wolfowitz, J. See Contributions to the theory of games, Feller, W.

Zelinsky, D. See van der Waerden, B. L.

Zygmund, A., Transue, W., Morse, M., Calder6n, A. P., and Bochner, S. Contributions to Fourier analysis. 163.

\section{INDEX OF INVITED ADDRESSES ${ }^{1}$}

Bing, R. H., 450.

Bourgin, D. G., 63.

Classes of transformations and bordering transformations, 223.

Bruck, R. H. An extension theory for a certain class of loops, 11.

van der Corput, J. G., 76

Erdélyi, A., 297.

The analytic theory of systems of partial differential equations, 339.

Federer, H., 260.

Fenchel, W. On the differential geometry of closed space curves, 44 .

Heins, M. H., 110.

Hopf, H., 174.

Huff, G. B., 250.

On the existence of plane curves with prescribed singularities, 411.

Loomis, L. H., 260.

McShane, E. J., 450.

Martin, M. H. Riemann's method and the problem of Cauchy, 238.

$\longrightarrow, 250$.

Montgomery, D., 450.

Mordell, L. J., 110.

Nakayama, T., 167.

Nehari, Z., 167.

- Bounded analytic functions, 354.

Niven, I., 367.

- The asymptotic density of sequences, 420.

Parker, W. V., Characteristic roots and field of values of a matrix, 103.

Rickart, C. E., 58.

Isomorphisms of infinite-dimensional analogues of the classical groups, 435 .

Segal, I. E., 283.

Smiley, M. F. Some questions concerning alternative rings, 36 .

Uhlenbeck, G. E., 110.

Walsh, J. L., 110.

Whitehead, G. W., 450.

Wiener, N. Problems of sensory prosthesis, 27.

1 Where title of address is not given, the reference is to the Report of the Meeting at which the address was given. 